\title{
Fluid-structure interaction phenomenon related to the expulsion and subsequent motion of multiple macroscopic particles
}

\author{
L. A. Florio \\ U.S. Army ARDEC, Picatinny Arsenal, NJ, USA
}

\begin{abstract}
Phenomena related to the expulsion and subsequent motion of multiple macroscopic particles are studied through numerical methods. Two basic systems are investigated: The first involves a set of particles set into motion in quiescent air and the second involves particle and free piston motion in the surroundings as the particles exit a flow channel under the influence of the gas driven piston motion. A fully coupled rigid body fluid structure interaction is utilized for up to a ten particle arrangement. The Navier-Stokes equations are solved for the in the fluid region with turbulence taken into account. The fluid induced pressure and viscous forces and moments as well as the effects of the interactions between the solid bodies are used in the calculations of the rigid body motion of the solid entities. A quaternion based kinematic formulation is implemented to prevent indeterminate system conditions. A soft collision model is applied to simulate the effects of the solid-body interactions. A moving and deforming fluid mesh facilitates the motion of the distinct bodies through the fluid with dynamic mesh adaption to ensure proper mesh size is maintained. With the modelling techniques developed, parametric studies were conducted to determine the influence of the particle size and density and the initial particle spacing and configuration on the paths the particles take and the spread of the particles after an equivalent time. The knowledge gained can improve the understanding of expelled particle motion and the means of controlling overall particle spread.
\end{abstract}

Keywords: computational fluid dynamics, macroscopic particle motion, fluid induced forces, soft collision model, quaternion kinematics. 


\section{Introduction}

The movement of multiple particles through a fluid can play a major role in the function of many systems or processes. Applications can range from providing a consistent dispersion of particulate matter for specific material properties, the transport of particles for pharmaceuticals, chemical and energy related industrial process, the motion of particles in space, and the flight of projectiles in defense systems. Understanding the fundamental fluid-rigid body interaction phenomenon that occur as objects move through a fluid and interact can provide the information and the guidance needed to improve or better control the desired system operation. This work reports on the development and results of a computational fluid dynamics based modelling technique to investigate the influence of key system parameters on the motion of multiple macroscopic particles through a compressible fluid in two major system configurations - free particle motion and ejection from a flow channel.

Previous work characterizing the motion of macroscopic particles through a fluid is extensive. The majority of the investigations have been carried out for viscous liquid working fluids as experimental measurements of the particle motion can be more readily captured. Some of the early works of fluid-particle motion include the studies by $\mathrm{Hu}$, Joseph, and Crotchet where a numerical technique to simulate the dropping of two circular particles in a fluid was described [1]. Lun and Bent [2] have developed an approach to numerically model the motion of multiple dilute frictional spheres in shear flow, obtaining important information regarding the conditions required for the formation of "microstructures" within the particle flow. Tsuji et al. [3] numerically investigated denser particle flows finding good correlation to the particle flow in experimental results for plug flows. Many of these early models used drag coefficients to estimate the fluid induced forces on the particles instead of the instantaneous fluid viscous and pressure forces based on the local flow field conditions. As computational capabilities have grown, more complex models, with more detailed computational grids and more detailed flow field results, have been used in determining the motion of the objects through the fluids. Among the more recent studies devoted mainly to numerical modelling of particle flow in a viscous liquid include the works of Wang et al. [4], Perrin and $\mathrm{Hu}$ [5], Shao et al. [6], and Apte et al. [7].

Integral to the development of the computational fluid dynamics models of the motion of the particles is the development of the techniques needed to incorporate the interaction between the moving particles. Various techniques of simulating the impact or collisions between two objects have been explored, most involving infinite cylinders or spheres as collision detection and directions normal and tangential to the impact can be readily calculated. Kruggel-Emden et al. [8], Deen et al. [9], and Dziugys and Peters [10] provide reviews of many of the most common collision models. Two major types of models exist. Hard collision models require calculations of exact impact times and generally rely on restitution coefficient data, with the coefficient found to be highly dependent upon velocity. The hard collision models generally consider binary collisions 
only and thus are typically used in dilute type systems. Soft collision models, which generally apply forces to simulate the effect of particle impact, can be used to investigate the simultaneous interaction between multiple objects or particles. A wide range of collisional force models has been developed based on a variety of theories, including molecular dynamics, hertz contact theory, and hysteresis in loading and unloading as described in references [8] through [10]. The hertz contact derived soft collision models are used in the current work. Variations of these models are also discussed by Brilliantov et al. [11], Di Renzo and Di Maio [12], and Norgaliev et al. [13].

While general modelling techniques to study the motion of particles through a fluid have been developed and used to investigate the related phenomenon, significant work into exploring the interaction, motion and spread of larger macroscopic particles, in general, and in high speed compressible flow, in particular, is limited [13]. In the current work, the relevant techniques found in the literature are applied, adapted, and modified to study the rigid body motion and interaction of macroscopic particles in high speed compressible flow. Direct coupling of the fluid flow, including the turbulence effects, and particle motion is implemented with a soft collision model accounting for the particle-particle interactions. Parametric studies in two major particle systems allow for the development of numerical modelling tools and the enhancement of the understanding of the phenomenon related to the motion and interaction of multiple macroscopic particles in compressible flow.

\section{Modeling methods}

The current study was conducted through extensive customization of a finite volume based computational fluid dynamics and heat transfer code, FLUENTC. The commercial code was used to exploit the existing moving mesh, remeshing and mesh adaption capabilities to simulate the rigid body motion such that the flow and solid body motion are directly coupled. The solution of the Navier Stokes, energy, and continuity equations, the inclusion of turbulence models, and the implementation of compressible liquid/volume of fluid models (for model validation case) and compressible gas material property models can be readily facilitated. However, extensive code development in the form of user defined functions was required to perform the kinematic analyses of the rigid bodies, to perform the collision detection, and to implement the collision effect models, as well as to output the necessary data.

\subsection{Kinematic model}

A quaternion based kinematic model was used to determine the motion of the moving rigid solid components. The method of quaternions is a more efficient means of describing the motion of a rigid body. The quaternion, $\mathrm{Q}$, developed by Hamilton, is essentially a four component vector. If $\bar{n}$ is a unit vector, the Euler axis, and $\alpha$ is a rotation or Euler angle about this axis, then the quaternion can be described in eqn. (1). 


$$
\mathrm{Q}=\left[\cos (\alpha / 2), n_{x} \sin (\alpha / 2), n_{y} \sin (\alpha / 2), n_{z} \sin (\alpha / 2)\right]
$$

The algebra involved with quaternions is simpler than the standard $\operatorname{roll}\left(\theta_{1}\right)$, $\operatorname{pitch}\left(\theta_{2}\right)$, and $\operatorname{yaw}\left(\theta_{3}\right)$ formulation with rotational matrices. The quaternions can be related to these standard angles as in eqn. (2).

$$
\left[\theta_{1}, \theta_{2}, \theta_{3}\right]=2\left[Q_{2}, Q_{3}, Q_{4}\right]
$$

Further details can be found in reference [10]. The quaternion formulation also can prevent ill determined systems that can arise upon calculation of these standard rotational angles. Particularly for the conditions studied in the current work with rotating macroscopic particles this formulation is of benefit.

\subsection{Collision effect model}

In the kinematic analyses performed, the forces and moments acting on the moving rigid bodies include the fluid induced forces as well as those forces that account for the interaction between the bodies. In the current model, a soft collision model based on the concepts described by Tsuji et al. [3] is employed with modifications derived from the work reported in [9] and [11-13]. Using this technique, all collisions are assumed to take place between circular or spherical objects. The method can be extended to non-circular/spherical objects by constructing a body from multiple overlapping circles/spheres. The collision detection and collision effect models then can be implemented between these circular/spherical components of the body. In doing so, care must be taken to ensure the proper positioning of the circles/sphere with respect to the center of gravity of the body is accounted for when determining the normal/tangential directions and distances, the total velocity at the contact position, and in the calculations of the moments caused by the collision effect forces.

The basic modelling technique simulates the collision effects through a normal and tangential visco-elastic non-linear spring and damper system. The collisional forces in the normal, $F_{n}$, and tangential, $F_{t}$, directions acting on particle 1, with forces equal in magnitude, but opposite in direction acting on particle 2, are activated when the value of $u$ defined in eqn. (3) becomes positive.

$$
u=R 1+R 2+\delta-d i s
$$

where $R 1$ and $R 2$ are the radii of particle 1 and 2 respectively as in fig. 1 and dis is the distance between the centers of the particles. $\delta$ is equal to the sum of the thickness of the boundary layer around each of the two colliding particles (BL1 and $B L 2$ respectively) and a small clearance spacing. The small clearance space ensures that the boundary layer meshes encapsulating and rigidly fixed to the particles do not overlap at collision. Hence, a small fluid clearance will remain between the colliding objects. The intent of the boundary layer is to better resolve the flow induced forces acting on the moving bodies as the remeshing method does not preserve the original size of the bounding cells. 


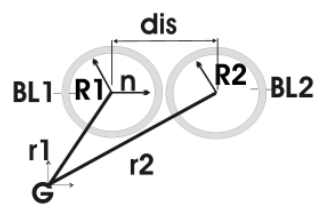

Figure 1: Collision between two circular/spherical objects.

As in fig. 1, a vector $\bar{r}$ leads from the center of particle 1 to the center of particle 2 , and $\bar{n}$ is the unit vector in this direction as in eqn. (4).

$$
\bar{r}=\overline{r_{2}}-\overline{r_{1}}, \quad \bar{n}=\bar{r} /|\bar{r}|
$$

The relative velocity at the contact position, $v r$, is given in eqn. (5), with $v$ representing the translational velocity, $\omega$ the rotation velocity, and $r_{c g}$ the vector from the center of gravity of the object to the contact position.

$$
\overline{v r}=\bar{v}_{2}-\bar{v}_{1}+R^{1 t o G}\left(\bar{\omega}^{1} \times \bar{r}_{c g 1}^{1}\right)-R^{2 t o G}\left(\bar{\omega}^{2} \times \bar{r}_{c g 2}^{2}\right)
$$

where the superscript 1 and 2 refers to the local coordinate system of particle 1 and 2 respectively and the notation $\mathrm{R}^{\mathrm{x}}$ to $\mathrm{y}$ is the transformation from the local coordinate system $\mathrm{x}$ to the global coordinate system $\mathrm{y}$. The normal component of the relative velocity can be defined as:

$$
\overline{v r}_{n}=(\overline{v r} \cdot \bar{n}) \bar{n}
$$

The tangential component of the relative velocity can be written as:

$$
\overline{v r}_{t}=\overline{v r}-\overline{v r}_{n}
$$

The tangential direction unit vector, $\bar{t}$, can then be determined from eqn. (7). With these parameters, following [12], the non-linear spring constants in the normal, $\mathrm{KN}$, and tangential, $\mathrm{KT}$, directions are given as:

$$
K N=(4 / 3) E^{*} \sqrt{R^{*}} \sqrt{u} ; K T=8\left[(2 / 3) G^{*} \sqrt{R^{*}} \sqrt{u}\right]
$$

Following [9], the damper coefficients in the normal, CN, and tangential, CT, directions, when CR (a normal restitution coefficient) or CRT (a tangential restitution) not equal to zero are :

$$
C N=\frac{\left(-2 \ln (C R) \sqrt{M^{*} K N}\right)}{\sqrt{\left(\pi^{2}+(\ln (C R))^{2}\right)}} \quad ; \quad C T=\frac{\left(-2 \ln (C R T) \sqrt{(2 / 7) M^{*} K T}\right)}{\sqrt{\left(\pi^{2}+(\ln (C R T))^{2}\right)}}
$$




$$
\begin{aligned}
\text { For } \mathrm{CR} \text { or } \mathrm{CRT} & =0 \\
\mathrm{CN} & =2 \sqrt{M^{*} K N} ; C T=2 \sqrt{(2 / 7) M^{*} K T}
\end{aligned}
$$

Following [12], subscript 1 refers to particle 1, subscript 2 to particle 2, E the Young's Modulus, $\mathrm{G}$ the bulk modulus, $\mathrm{M}$ the mass, and $\vartheta$ the Poisson's Ratio :

$$
\begin{aligned}
E^{*}=\left[\left(1-\vartheta_{1}^{2}\right) / E_{1}+\left(1-\vartheta_{2}^{2}\right) / E_{2}\right]^{-1} ; R^{*} & =\left[1 / R_{1}+1 / R_{2}\right]^{-1} \\
G^{*}=\left[\left(2-\vartheta_{1}\right) / G_{1}+\left(2-\vartheta_{2}\right) / G_{2}\right]^{-1} ; M^{*} & =\left[1 / M_{1}+1 / M_{2}\right]^{-1}
\end{aligned}
$$

The forces acting on particle 1 can then be formulated as :

$$
\begin{gathered}
\overline{F N}=-(K N u) \bar{n}-C N \overline{v n} \\
\text { If }|\overline{F T}| \leq \mu|\overline{F N}| \overline{\mathrm{FT}}=-(K T \overline{d r})-C T \overline{v t}, \\
\text { If }|\overline{F T}|>\mu|\overline{F N}| \overline{\mathrm{FT}}=-\mu|\overline{F N}| \overline{\mathrm{t}}
\end{gathered}
$$

with $\overline{d r}=\int \overline{v t} d t$ integrated from start of collision

Equal but oppositely directed forces act on particle 2 . The total forces and related moments due to these collisional effect forces and the direct fluid induced forces must be determined and summed to estimate the motion of each of the rigid bodies involved.

\subsection{Numerical procedure}

The modelling technique algorithm is summarized in the flow chart in fig. 2 .

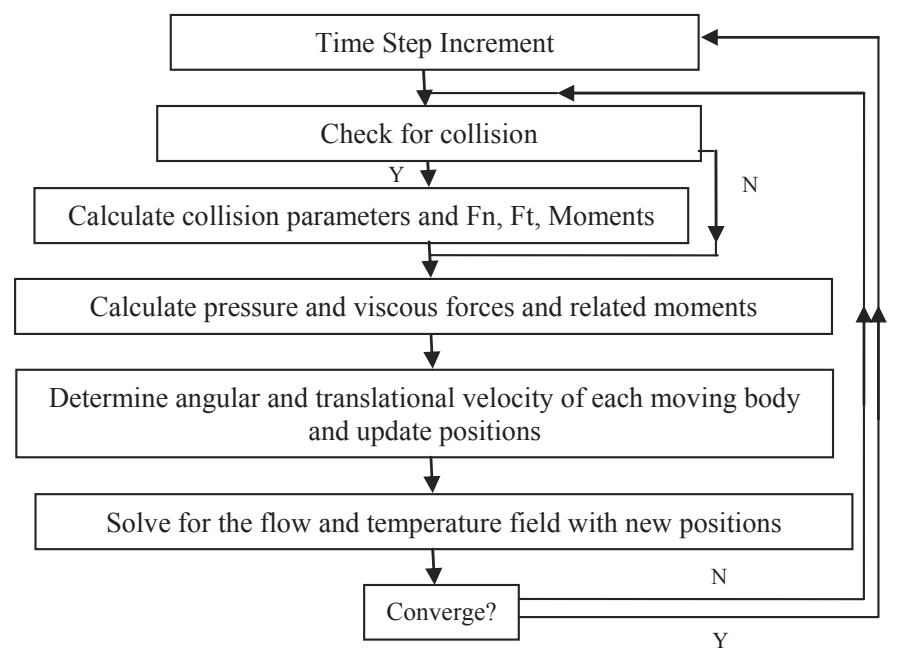

Figure 2: Algorithm flow chart.

A realizable $\kappa-\varepsilon$ turbulence model is employed with an implicit solution method in time and space. PISO pressure-velocity coupling is implemented with 
QUICK spatial discretization for the momentum and energy equations and for the density solution. Body force weighted discretization is used for the pressure. Typical time steps of $1 \mathrm{e}-08 \mathrm{~s}$ are used. An ideal gas is assumed for air with temperature varying specific heat, viscosity, and thermal conductivity. Mesh adaption and refinement based on pressure gradients is used to control the mesh size and to assure low mesh sensitivity. Mesh sensitivity studies for the boundary layer size as well as for the remeshing (moving) and mesh adaption related parameters were performed for a representative case. Thirty-two processors on a 64 bit DELL Linux cluster system were used to execute the analyses.

\subsection{Model Validation}

The modelling techniques were validated by comparisons to published data involving particles dropping through a viscous liquid. A volume of fluid approach was used with air above the liquid column. Good correlation to the published results can be observed in fig. 3. Fig. 3a involves a single particle or cylinder dropping in a column for two different fluids. Fig. $3 \mathrm{~b}$ involves the motion of a large and small particle with the large particle released above the small particle. The discrete data points in the figures represent data from published studies while the continuous curves are the current model results.

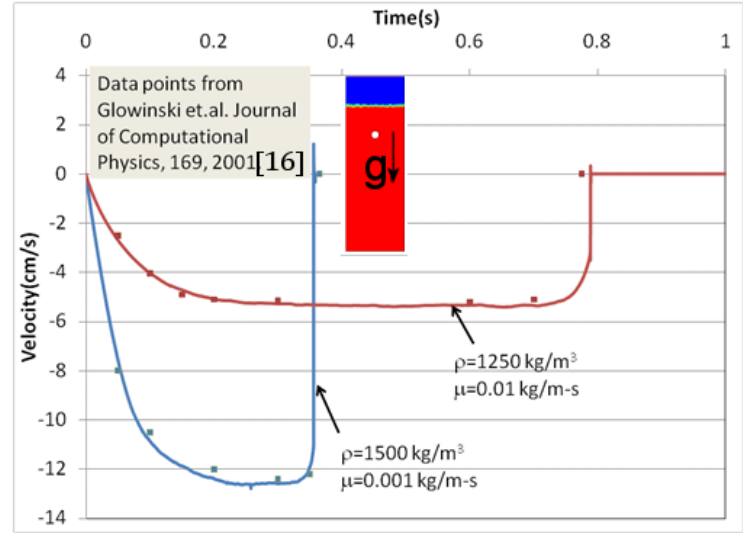

(a)

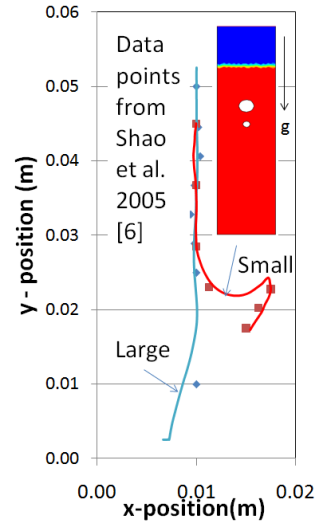

(b)

Figure 3: Comparison to published results a) velocity of single particle dropping in channel [16] b) trajectory of two particles dropping in a channel [6].

\section{Problem statement}

The modelling method described above is then implemented to investigate the initial motion of long infinite cylinders through air. In order to gain insight into the relationships between key system parameters and the particle motion characteristics, two basic systems are studied in this investigation. The first is the free motion of circular particles given an initial velocity in the positive $\mathrm{x}$ 
direction in initially quiescent air. Gravity is acting in the negative y direction. For this system type, two different configurations are studied. The first is a nine equi-size, equi-density inline particle array as in fig. 4a. The second is a ten equi-size, equi-density offset particle array as in fig. 4b. Parametric studies varying the initial spacing distance, the particle, or cylinder, diameter, and the particle density were performed. The cases investigated involved densities of $\rho / \rho_{\text {nom }}$ of $0.5,1$, and 2 , cylinder radii of $R / R_{\text {nom }}$ of $0.5,1$, and 2 , and initial spacing of $\mathrm{b} / \mathrm{D}$ of $1.25,1.5,2.0$, and 3.0 with $\mathrm{a}=\mathrm{b}=2 \mathrm{c}$. The subscript "nom" denotes the nominal density or radius. In each arrangement, a large fluid domain of approximately 150 cylinder diameters surrounds the area shown in fig. 4 with pressure outlet boundary conditions applied. D denotes the particle diameter.

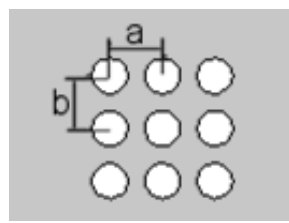

a)

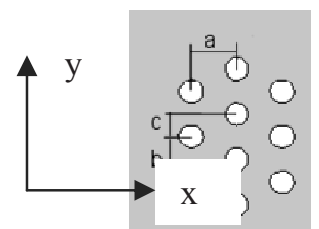

b)

Figure 4: System configurations for particles released into quiescent air a) 9 particle inline array; b) 10 particle offset array.

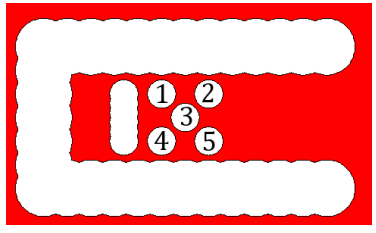

Figure 5: System for study of particle motion and expulsion from a flow channel.

The second major system investigated is a flow channel containing a moving free piston/plate and 5 infinite cylindrical particles as in fig. 5. Overlapping cylinders are used to define the non-circular components so that essentially the same collision detection and effect models can be utilized. The infinite flow channel in fig. 5 consists of 31 cylinders and the piston plate is formed of 6 cylinders. Five particles of equal size and density are placed ahead of the piston. The fluid behind the plate is supplied an elevated temperature and pressure and the plate is given an initial $x$ velocity. The plate-particle interaction initiates the particle motion. The characteristics of the particle motion for 5 different ratios of the moving plate mass to the single particle mass were examined in the parametric studies performed. The system components in fig. 5 are surrounded by a large fluid zone as in the previous case. 


\section{Results}

\subsection{Free particle motion through initially quiescent air}

While the motion of a particle may deviate from its initial path due to vortex shedding and the influences of gravity, the results of the parametric studies for the systems in fig. 4 have shown that for a group of macroscopic particles, significant deviation from an initial trajectory develops only when the surrounding particles are sufficiently close to affect the flow conditions moving over nearby particles. Further information regarding the influence of the particle size, density, and initial spacing on the motion of particles instantaneously given a horizontal velocity in initially quiescent air will be briefly summarized below.

As the set of particles moves through a fluid, the presence of neighboring particles changes the flow patterns and pressures of the nearby gas. The particles on the exterior of the particle pack tend to experience larger pressure differences along a given particle surface due to the higher fluid velocities moving over the portions of the surfaces external to the particle pack and to a lesser extent the high velocity flows in the passageways between the particles. The particle spread initiates as the pressure difference along the particle surface pushes the exterior particles away from the neighboring particles. The particles in the wake of leading particles and in the interior of the particle group are surrounded by lower velocity flows, are "protected" from the higher pressure differences, and exhibit little deviation from their initial trajectory.

Fig. 6 illustrates the characteristic trends in the variation in the spread with the initial particle spacing to diameter ratio, $b / \mathrm{D}$, for various particle radii and densities. The plots depict the ratio of the y-directed particle spread, $\Delta \mathrm{sp}$, at $1 \mathrm{~ms}$ from release to the initial y-directed spread. The spread is defined as the maximum distance in the y direction between the centers of any particles in the system. The initial spread, $\Delta$ spinit, is $2 \mathrm{~b}$ for the 9 particle inline configuration and $3 \mathrm{~b}$ for the 10 particle offset configuration. The results indicate the initial spacing to diameter ratio has a strong influence on the particle spread. As the particles become more closely spaced, the surrounding particles have a greater effect on the local flow conditions at neighboring particles, leading to greater $y$ directed force imbalances and the greater the spread. At a b/D ratio of 3, the particles are far enough apart that the flow conditions at a given particle are only minimally affected by the presence of the surrounding particles and the spread of particle pack is minimal for the majority of the cases studied (fig. 6). The results presented also demonstrate that the particle spread increases with a decrease in the particle size as a result of the smaller particle mass and greater particle acceleration. Additionally, the particle spread increases with a decrease in the particle material density. The spread conditions are more sensitive to changes in the particle size than the particle density. For the same $R / R_{\text {nom }}$ and $\rho / \rho_{\text {nom }}$ ratio, the particle size change affects the basic flow structures and results in a change in mass of the order of $\left(\mathrm{R} / \mathrm{R}_{\text {nom }}\right)^{2}$ while the particle density change simply produces a mass change of $\left(\rho / \rho_{\text {nom }}\right)$. Fig. 7 provides representative plots of the 

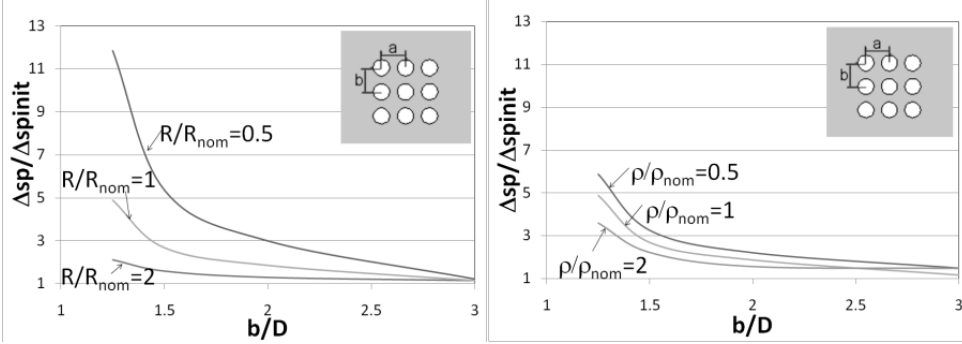

a)
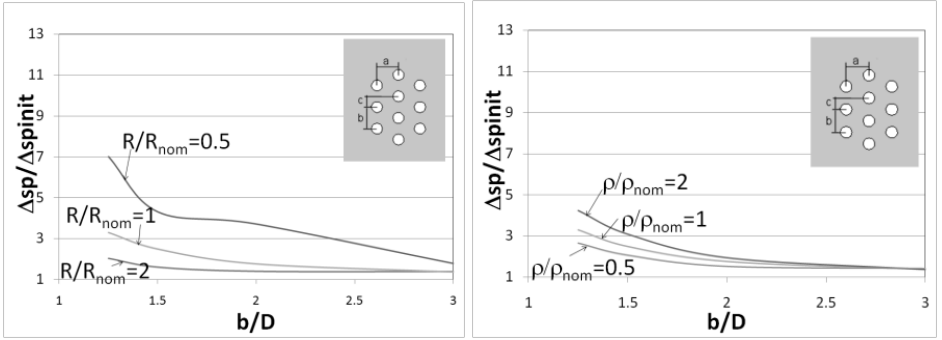

b)

Figure 6: Particle spread increase as a function of initial particle spacing to diameter ratio for the various particle size and density conditions indicated: a) Inline configuration; b) Offset configuration.

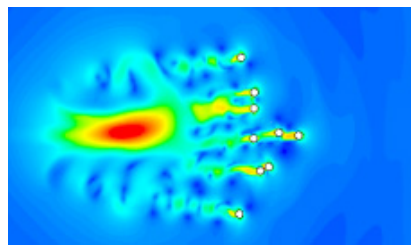

a)

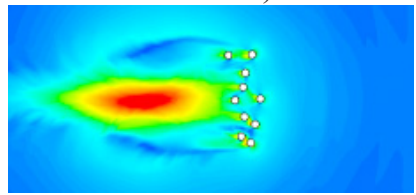

d)

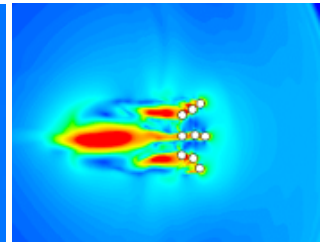

c)

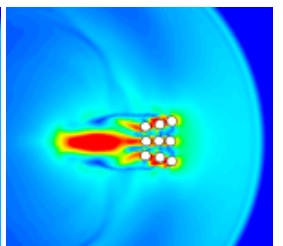

b)

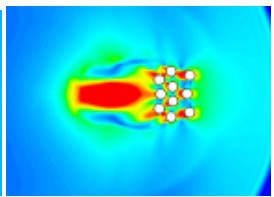

e) f)

Figure 7: Velocity field contour plots at $1 \mathrm{~ms}$ for $\rho / \rho$ nom $=1 \mathrm{~b} / \mathrm{D}=1.25$ a) $R / R_{\text {nom }}=0.5$ inline; b) $R / R_{\text {nom }}=1.0$ inline; c) $R / R_{\text {nom }}=2.0$ inline; d) $R / R_{\text {nom }}=0.5$ offset; e) $R / R_{\text {nom }}=1.0$ offset; f) $R / R_{\text {nom }}=2.0$ offset.

velocity contours and particle positions resulting for various particle radii for the inline and offset cases for one initial particle spacing. Similar, though less pronounced, trends are observed for variations in the particle density. 


\subsection{Particle motion exiting a flow channel}

The modelling techniques are next applied to the study of the motion of particles expelled from a flow channel utilizing the system described in fig. 5 where a plate and pressurized gas are used to initiate the motion of the particles at rest. The parametric study of the effect of the plate mass to single particle mass ratio indicates that the particle interaction and its impact on the y spread is much more complex than for the free particle motion conditions due to the greater level of interaction (collisions) between the solid components of the system. The particle spread was found to be a function of the positioning, orientation, and velocity of the particles prior to collision, the location and timing of the collisions, the path the particles take after collision, the location of neighboring particles, and the local flow conditions, all interdependent and highly transient conditions.

Due to the high level of dependency on the instantaneous particle and solid body interactions, no clear trend in the particle spread as a function of the mass ratio was detected from the parametric study results, as seen in fig. $8 \mathrm{a}$. The trajectory of particle 5 , as labelled in fig. 5 , is shown in fig. $8 \mathrm{~b}$, indicating the complex nature of the motion of the particles upon channel exit. Particle collisions prior to and just after exit exhibit a great influence on the particle spread. These collisions direct the positioning and trajectory of the particles as they begin to move into the surroundings and also direct the gas flow patterns which in turn impact the particle motion. Fig. 9 provides an illustrative comparison showing the sensitivity of the y-spread to collision timing and particle positioning. For Mplate /Mparticle of 24 (fig. 9a), particles 2 and 5 (fig. 5) collide and are directed such that their velocity in the y direction is minimal. For Mplate/Mparticle equal to 18 (fig. 9b), particle 5 collides with the lower wall, bounces back, moves past particle 2 without collision, and is caught in the high speed exiting flow. Hence, the motion of the particles in the arrangement in fig. 5 is closely tied to the local flow conditions, the time-history of the particle collisions, and the positioning of the neighboring particles and bodies.

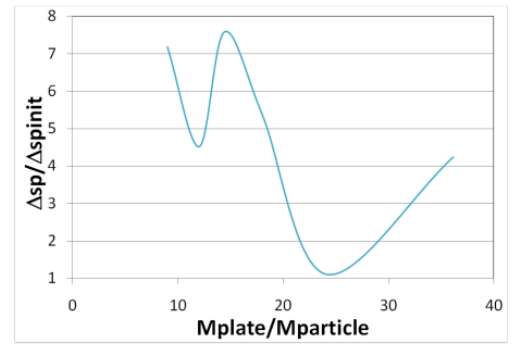

a)

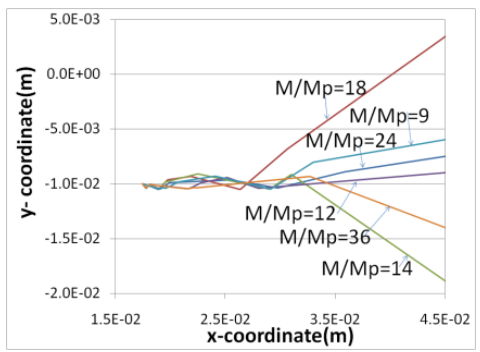

b)

Figure 8: Typical expulsion data a) Ratio of $y$-directed spread ( $\Delta \mathrm{sp}$ ) to initial $\operatorname{spread}(\Delta$ spinit $)$ as a function of plate-particle mass ratio b) Trajectory of particle 5 (fig. 5) for plate-particle mass ratio (M/Mp) cases indicated. 

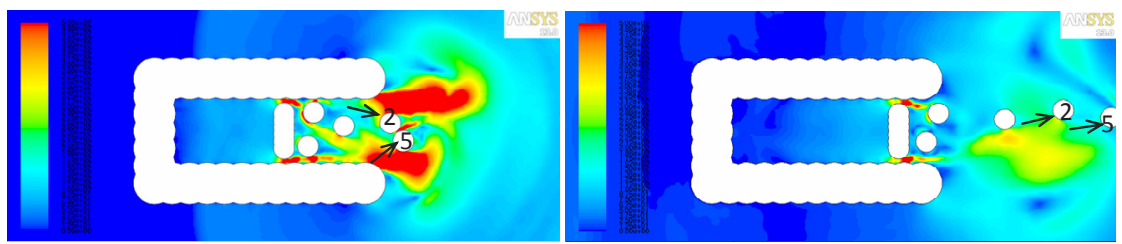

a)
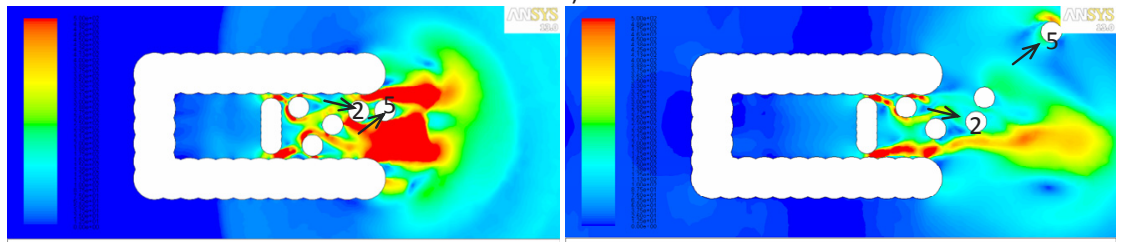

b)

Figure 9: Representative velocity fields depicting exit conditions for particles 2 and 5 (fig.
Mplate/Mparticle $=18$.

5) for:

a) Mplate/Mparticle $=24$

\section{Conclusions}

In this work a method for simulating the coupled fluid flow and motion and interaction of multiple rigid bodies was presented and was shown capable of predicting the characteristics of the rigid body motion and fluid flow. During unobstructed, free particle motion, the y-directed spread was found to be greater for closer initial particle spacing, for smaller particles, and for particles of lower density. The particles must be sufficiently close to one another in order to initiate significant particle spread. The particle spread conditions are more sensitive to the particle size than the particle density. Studies of particle expulsion from a flow channel indicate the exiting particle spread is a complex function of the interactions between the flow field, particles, plate, and channel and is highly affected by the instantaneous collision occurrences and collision history, the position and motion of the colliding and nearby objects, and the local gas flow.

This work is part of an ongoing study. In addition to further parametric studies and increased particles counts and particle configurations, areas currently being explored include refinements of the modelling techniques to simulate the collisions between non-circular bodies. The effects of using various particle collision models are also being investigated towards the development of collision models appropriate for high speed compressible flows.

\section{Acknowledgement}

This work was funded by an ARDEC In-House Laboratory Independent Research Project. 


\section{References}

[1] Hu, H.H., Joseph, D.D., and Crochet, M.J., Direct numerical simulation of fluid particle motions. Theoretical and Computational Physics, 3, pp. 285306, 1992.

[2] Lun, C.K.K. and Bent, A.A., Numerical simulation of inelastic frictional spheres in simple shear flow, Journal of Fluid Mechanics, 258, pp.335-353, 1994.

[3] Tsuji, Y, Tankada, T., and Ishida, T., Lagrangian numerical simulation of plug flow of cohesionless particles in a horizontal pipe, Powder Technology, 71, pp.239-250, 1992.

[4] Wang, Z., Fan, J., and Luo, K. Combined multi-direct forcing and immersed boundary method for simulating flows with moving particles, International journal of multiphase flow, 34, pp.283-302, 2008.

[5] Perrin. A., and Hu, H.H., An explicit finite-difference scheme for simulation of moving particles, Journal of Computational Physics, 212, pp. 166-187, 2006.

[6] Shao, X.-M., Liu, Y., and Yu, Z.-S., Interactions between two sedimenting particles with different sizes, Applied Mathematics, and Mechanic, 26(3), pp. 407-414, 2006.

[7] Apte, S., Martin, M., and Patankar, N.A., A numerical method for fully resolved simulation (FRS) of rigid particle-flow interactions in complex flows, Journal of Computational Physics, 228, pp. 2712-2738, 2009.

[8] Kruggel-Emden, H. et al., Review and extension of normal force models for the discrete element method., Powder Technology, 171, pp. 157-173, 2007.

[9] Deen, N.G. et al. Review of discrete particle modelling of fluidized beds, Chemical Engineering Science, 62, pp. 28-44, 2007.

[10] Dziugys, A. and Peters, B. An approach to simulate the motion of spherical and non-spherical fuel particles in combustion chambers, Granular Matter, 3, pp. 231-265, 2001.

[11] Brilliantov, N.V. et al., Model for collisions of granular gases, Physical Review E, 53(5), pp. 5382-5392, 1996.

[12] Di Renzo, A. and Di Maio, F.P. An improved integral non-linear model for the contact of particles in distinct element simulations, Chemical Engineering Science, 60, pp. 1303-1312, 2005.

[13] Norgaliev, R.R., Dihn, T.N., Theoganous, T.G., On modelling of collisions in direct numerical simulation of high-speed multiphase flows. Proceedings of the Third International Conference on Computational Fluid Dynamics FDICCFD' 3 Toronto 12-16 July 2004.

[14] Ickes, B.P., A new method for performing digital control system attitude computations using quaternions, 8(1), AIAA Journal, pp. 13-17, 1970.

[15] Kodam, M. et al., Force model considerations for glued-sphere discrete element method simulations. Chemical Engineering Science, 64, pp. 34663475, 2009. 
[16] Glowinski, R. et al. A fictitious domain approach to the direct numerical simulation of incompressible viscous flow past moving rigid bodies: Application to particulate flow, Journal of Computational Physics, 169, pp. 363-426, 2001. 